\title{
Pesquería y biología de Rhinobatos percellens (Rajiformes: Rhinobatidae) capturados por la pesquería artesanal de playa La Pared, Venezuela
}

\author{
Alejandro Tagliafico ${ }^{1}$, Néstor Rago ${ }^{2}$ \& María Salomé Rangel ${ }^{1,3}$ \\ 1. Escuela de Ciencias Aplicadas del Mar, Núcleo de Nueva Esparta, Universidad de Oriente, Boca de Río, 6304, Isla de \\ Margarita, Venezuela; tagliaficoa@gmail.com \\ 2. Oceanografía y pesca, Fundación de Ciencias Naturales La Salle, Isla de Margarita Venezuela; nestorrago@yahoo.es \\ 3. Área de Biología, Departamento de Ciencias, Núcleo de Nueva Esparta, Universidad de Oriente, Guatamare, 6301, \\ Isla de Margarita; salome453@gmail.com
}

Recibido 23-I-2012. Corregido 06-VI-2012. Aceptado 06-VII-2012.

\begin{abstract}
Fishery and biology of Rhinobatos percellens (Rajiformes: Rhinobatidae) caught by the artisanal fishery at La Pared beach, Venezuela. $R$. percellens is incidentally caught in Venezuela by the artisanal fishery using bottom gillnets. This species was classified by the IUCN as being "near threatened", and there is poor knowledge about its biology and fishery in Venezuela. For this reason, we analyzed the specimens caught by the artisanal fleet in playa La Pared, once a week, from January to December 2007. We determined total length, sex and maturity for each captured specimen. A total of 210 specimens were analyzed, 159 females and 51 males. The $81 \%$ of all specimens caught were adults, but within this group $27 \%$ were pregnant. The average size of sexual maturity was found between 51 and $52 \mathrm{~cm}$ for females and males, respectively. Adult females were found all year round, and pregnant females in seven of the months sampled; highest numbers were found between February-March and September. The analyzed females had a total of 96 embryos with a size range between 2 and $19 \mathrm{~cm}$, with a maximum fertility of four embryos per litter. February and June-July showed the maximum breeding time, and considering the broad range of embryos sizes and the frequency of pregnant females along the different months, allow us to believe that they reproduce throughout the year. Fishing regulation including minimum catch size in relation to maturity length and release of pregnant females in water is recommended. Rev. Biol. Trop. 61 (1): 149-160. Epub 2013 March 01.
\end{abstract}

Key words: Elasmobranchii, Batoidea, Guitarfish, Caribbean.

Rhinobatos percellens Link 1790, es una raya de cuerpo alargado $(<1 \mathrm{~m})$ pero no muy ancho, aplanada dorsoventralmente y con hábitos demersales. Se distribuye desde el Mar Caribe hasta el norte de Argentina, siendo una especie común en fondos someros de la plataforma nororiental venezolana (Cervigón \& Alcalá 1999). En esta zona, la especie forma parte de la fauna acompañante en la pesca con redes de fondo, ya que no hay una pesquería dirigida a sus capturas. Históricamente $R$. percellens ha sido poco consumida, e incluso a veces desechada (Cervigón \& Alcalá 1999). Actualmente, con la disminución de otros recursos pesqueros, la especie está adquiriendo mayor interés comercial, y se puede encontrar de manera constante en diversos mercados de la zona.

Esta especie se alimenta principalmente de crustáceos y en menor proporción de peces (Polo-Silva \& Grijalba-Bendeck 2008, Bornatowsky et al. 2010). Tiene una reproducción vivípara aplacentaria (Grijalba-Bendeck et al. 2008). Se caracteriza por presentar baja resiliencia, su población es capaz de duplicarse en un tiempo mínimo de 4.5-14 años (Froese et al. 2002), y su vulnerabilidad es considerada de moderada a alta (Cheung et al. 2005). Ha sido 
incluida en la categoría "Casi Amenazada" por la IUCN, debido a que está sometida constantemente a altos niveles de explotación pesquera en sus zonas de distribución (Casper \& Burgess 2004). En Brasil, una especie de características biológicas similares (Rhinobatos horkelii) ha sido catalogada en peligro crítico, debido a severas disminuciones poblacionales $(>80 \%$ entre 1986-2001) producto de la sobrepesca (Lessa \& Vooren 2007), lo que ha llevado a inferir, a causa de la similitud entre las pesquerías de ambos países, que las poblaciones de $R$. percellens también han disminuido; aunque es necesario recaudar datos sobres sus capturas para confirmarlo (Casper \& Burgess 2004).

En Venezuela, sólo existen estudios sobre taxonomía y algunos datos de las capturas de R. percellens (Cervigón \& Alcalá 1999, Marval \& Cervigón 2009). Actualmente, no existe ninguna regulación que controle sus capturas. Sin embargo, la prohibición total de las redes de arrastre industrial (Art. 23, Ley de Pesca y Acuicultura 2008), es una medida que podría contribuir con la conservación de la especie.

El objetivo del presente trabajo es aportar información sobre aspectos reproductivos de $R$. percellens, y describir algunas características de su pesquería, para contribuir a su conocimiento y orientación hacia un correcto manejo pesquero.

\section{MATERIALES Y MÉTODOS}

Área de estudio: Playa La Pared es una pequeña comunidad pesquera ubicada en la isla de Margarita, península de Macanao, estado Nueva Esparta, región nororiental de Venezuela $\left(11^{\circ} 03^{\prime} 32.53^{\prime \prime} \mathrm{N}-64^{\circ} 18^{\prime} 47.25^{\prime}\right.$ W). Es una zona influenciada por fuertes vientos alisios que ocasionan el fenómeno de surgencia costera. La temperatura superficial del agua varía a lo largo del año entre $22-27^{\circ} \mathrm{C}$ y la salinidad superficial entre 34-36.25ppm (Aparicio 2003). El régimen de precipitación se caracteriza por dos períodos lluviosos (de junio a agosto y de diciembre a febrero) y dos secos (marzo a mayo y septiembre a noviembre) (Leal 2008).
Playa La Pared, posee una flota pesquera artesanal de unas 12 embarcaciones de madera, de entre cinco y nueve metros de eslora, propulsadas con motores fuera de borda a gasolina (dos tiempos). Utilizan diversos artes de pesca dependiendo de la época y abundancia de los recursos pesqueros.

Para este estudio, se realizaron muestreos una vez por semana, en el período enerodiciembre de 2007. Los ejemplares provinieron de los desembarques de redes de enmalle de fondo o filetes fonderos, con una abertura de malla ubicada entre tres y siete pulgadas, un alto de entre dos y siete metros, y con una longitud de 200-600m. Descripciones detalladas de las embarcaciones y artes de pesca, son reseñadas por Iriarte (1997) y González et al. (2006). Posteriormente, las especies fueron identificadas utilizando las claves taxonómicas de Cervigón \& Alcalá (1999). Especímenes de referencia fueron catalogados y conservados en la colección del Laboratorio de Ictiología, Escuela de Ciencias Aplicadas del Mar (CIECAM) de la Universidad de Oriente.

Estructura de talla y Captura por Unidad de Esfuerzo (CPUE): Utilizando una regla de $1 \mathrm{~mm}$ de precisión, se tomó la longitud total (Lt) de cada ejemplar, distancia comprendida entre la punta del hocico y extremo posterior de la aleta caudal. Se determinaron las frecuencias de longitudes por intervalos de talla para machos y hembras por separado. Se compararon las longitudes promedio de ambos sexos mediante ANDEVA de una vía.

Cada individuo capturado por bote fue contado y pesado con una balanza de campo de $1 \mathrm{~g}$ de precisión. Además, se contabilizó el número de botes con red de enmalle de fondo en la plataforma continental por día (excluyendo las faenas provenientes del talud continental, por ser zonas profundas donde la especie no forma parte de las capturas). El índice de abundancia relativa, Captura por Unidad de Esfuerzo (CPUE) fue definido como el número de individuos capturado por faena de pesca (ind./faena) y kilogramos de captura por faena de pesca $(\mathrm{kg} /$ faena). 
Aspectos reproductivos: Los sexos se diferenciaron por la presencia del órgano copulador masculino (claspers o mixopterigios) en los machos y su ausencia en las hembras. Para evaluar diferencias significativas en la proporción sexual anual y mensual, se aplicó la prueba del Chi-cuadrado $\left(\chi^{2}\right)$. Adicionalmente, se calcularon las proporciones sexuales mensuales tomando en cuenta únicamente los individuos maduros para indagar sobre posibles épocas de apareamiento.

Los organismos capturados por cada embarcación fueron analizados separadamente, estableciendo diferentes grupos en función de las combinaciones de sexos y estadios de madurez sexual, con el fin de indagar sobre la frecuencia de posibles grupos sociales.

Los machos maduros fueron identificados cuando los claspers estaban completamente desarrollados, presentaban fuerte calcificación, fácil rotación en la base, extremo distal extensible (Conrath 2004) y presencia de fluido seminal (Bizzarro et al. 2007).

La madurez de las hembras fue identificada a través de la observación macroscópica del aparato reproductivo. Dependiendo de la presencia o ausencia de ovocitos bien definidos con acumulación de yema de color amarillenta, fueron consideradas maduras o inmaduras, respectivamente (Conrath 2004). En los casos en que los pescadores lo permitieron, se contó y midió el diámetro de ovocitos. Las hembras grávidas fueron reconocidas por presentar embriones en sus úteros. Los embriones fueron medidos y contados, para determinar la talla cercana al nacimiento y la fecundidad uterina (Conrath 2004).

Se calculó la talla media de madurez sexual para ambos sexos por separado y en conjunto, utilizando el software $R$, mediante el método de mínimos cuadrados, para lo cual se determinó la proporción de organismos maduros para cada intervalo de talla, y se estimó la proporción de maduros predicha para cada talla mediante la función logística:

$$
M_{f}=\frac{1}{1+e^{-a\left(L_{i}-b\right)}}
$$

donde $M_{f}$ es la fracción de individuos maduros; "a" y "b" son parámetros a ser estimados. El parámetro "a" representa el cambio en la pendiente de $\mathrm{M}_{\mathrm{f}}$ como función de la longitud $\left(\mathrm{L}_{\mathrm{i}}\right)$, y "b" representa la longitud de madurez del 50\% de los organismos maduros $\left(\mathrm{L}_{50}\right)$.

Se determinó la frecuencia porcentual mensual de cada una de las diferentes etapas de madurez observadas para machos y hembras, con la finalidad de observar posibles picos en las etapas reproductivas. Adicionalmente, estas frecuencias fueron comparadas con las tallas de los embriones para verificar el período de alumbramiento y de gestación.

\section{RESULTADOS}

Estructura de talla y CPUE: Un total de 210 ejemplares de $R$. percellens fueron analizados, presentaron una longitud entre $30-76.8 \mathrm{~cm}$, con un promedio y desviación estándar de $61.4 \pm 7.2 \mathrm{~cm}$. Las hembras $(63 \pm 6.2 \mathrm{~cm})$ presentaron longitudes promedio superiores a los machos $(56.2 \pm 9 \mathrm{~cm})$ (Fig. 1) evidenciándose una diferencia significativa entre las longitudes de ambos sexos (ANDEVA; $F_{(1.208)}=32.06$, $\mathrm{p}<0.0001)$.

Las CPUE promedios quedaron establecidas en $2.7 \mathrm{ind}$./faena y $2.5 \mathrm{~kg} /$ faena. Los mayores valores se registraron en el segundo semestre del año (Fig. 2). El peso total varió entre 323-1729g, con un promedio general de 870 g (Fig. 3A). El número máximo de individuos capturados en una misma faena fue 13 , sin embargo, en el $45 \%$ de las faenas no se capturó ningún ejemplar. El promedio de capturas mensual estuvo alrededor de $16 \mathrm{~kg}$, con un máximo de $40 \mathrm{~kg}$ en septiembre (Fig. 3B).

Para los meses de abril y noviembre no se registró ningún ejemplar debido a que los pescadores destinaron el esfuerzo de pesca a recursos pelágicos y a otras áreas de mayor profundidad donde la especie no habita.

Aspectos reproductivos: De 210 individuos analizados, 159 fueron hembras (76\%) y 51 fueron machos $(24 \%)$ lo que indica una diferencia significativa en la proporción sexual, equivalente a unas tres hembras por cada macho 


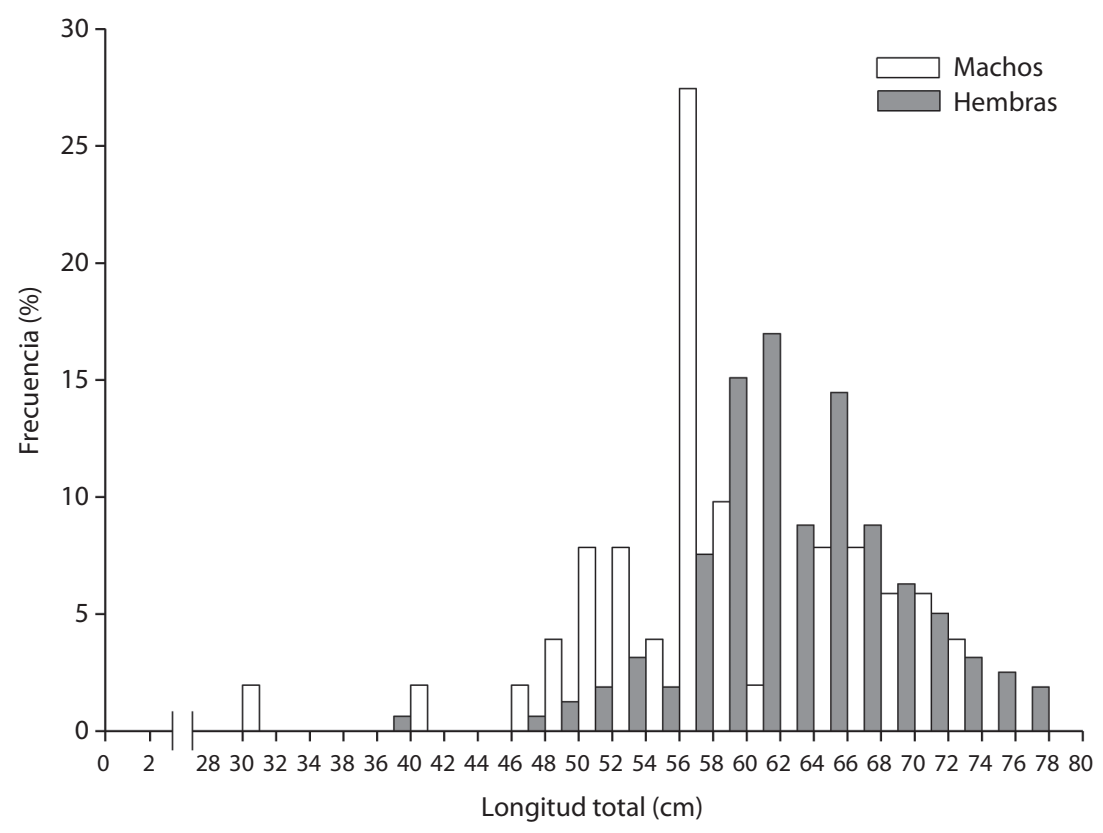

Fig. 1. Estructura de talla de Rhinobatos percellens capturados por la pesquería artesanal de playa La Pared, Venezuela. Fig. 1. Length structure of Rhinobatos percellens caught by the artisanal fishery of playa La Pared, Venezuela.

$\left(\chi^{2}=63.9, \mathrm{p}<0.05\right)$. Sin embargo, al analizar la proporción sexual de cada mes, se encontraron diferencias en todos los meses excepto en enero, marzo, mayo y julio. No obstante, al comparar las proporciones sexuales únicamente tomando en cuenta ejemplares maduros, solo se encuentran proporciones similares en enero, julio y septiembre. Además se observó que entre febrero-mayo no hubo machos maduros y en agosto, octubre y diciembre su presencia fue escasa; en cambio, las hembras maduras predominaron prácticamente todo el periodo de estudio excepto en junio, donde los machos representaron el 75\% (Fig. 4).

Analizando las capturas de cada bote por separado, se detectaron 15 posibles grupos sociales con diferentes combinaciones de sexos y estadios de madurez, destacando los siguientes grupos: hembras grávidas solitarias; hembras maduras solitarias; y machos inmaduros acompañados por hembras maduras $(14 \%$ en todas estas categorías). Entre otras combinaciones destacaron con un 7\%: machos inmaduros con hembras inmaduras; machos maduros solitarios; machos maduros con hembras maduras; y un grupo mixto de machos inmaduros acompañados por hembras inmaduras, maduras y grávidas. Otras ocho posibles combinaciones observadas en las capturas tuvieron proporciones menores a $4 \%$.

La hembra madura de menor tamaño fue un ejemplar de $48.7 \mathrm{~cm}$ en estado de gravidez, pero solo a partir de $58 \mathrm{~cm}$ todas las hembras se encuentran maduras. El macho maduro de menor tamaño fue de $49 \mathrm{~cm}$ y a partir de $60 \mathrm{~cm}$, todos se encontraban maduros. La longitud promedio para la madurez sexual $\left(\mathrm{L}_{50}\right)$ de las hembras fue de $51 \mathrm{~cm}$, ligeramente menor que los machos $(52 \mathrm{~cm})$, aunque no muestran diferencias (tomando los intervalos de confianza como criterio), por lo cual se estimó un $\mathrm{L}_{50}$ para ambos sexos de $49 \mathrm{~cm}$ (Cuadro 1).

El diámetro máximo de los ovocitos varió entre $0.2-4.3 \mathrm{~cm}$; este último, fue observado en una hembra grávida con cuatro embriones de longitudes entre $5-6 \mathrm{~cm}$. Otra hembra 

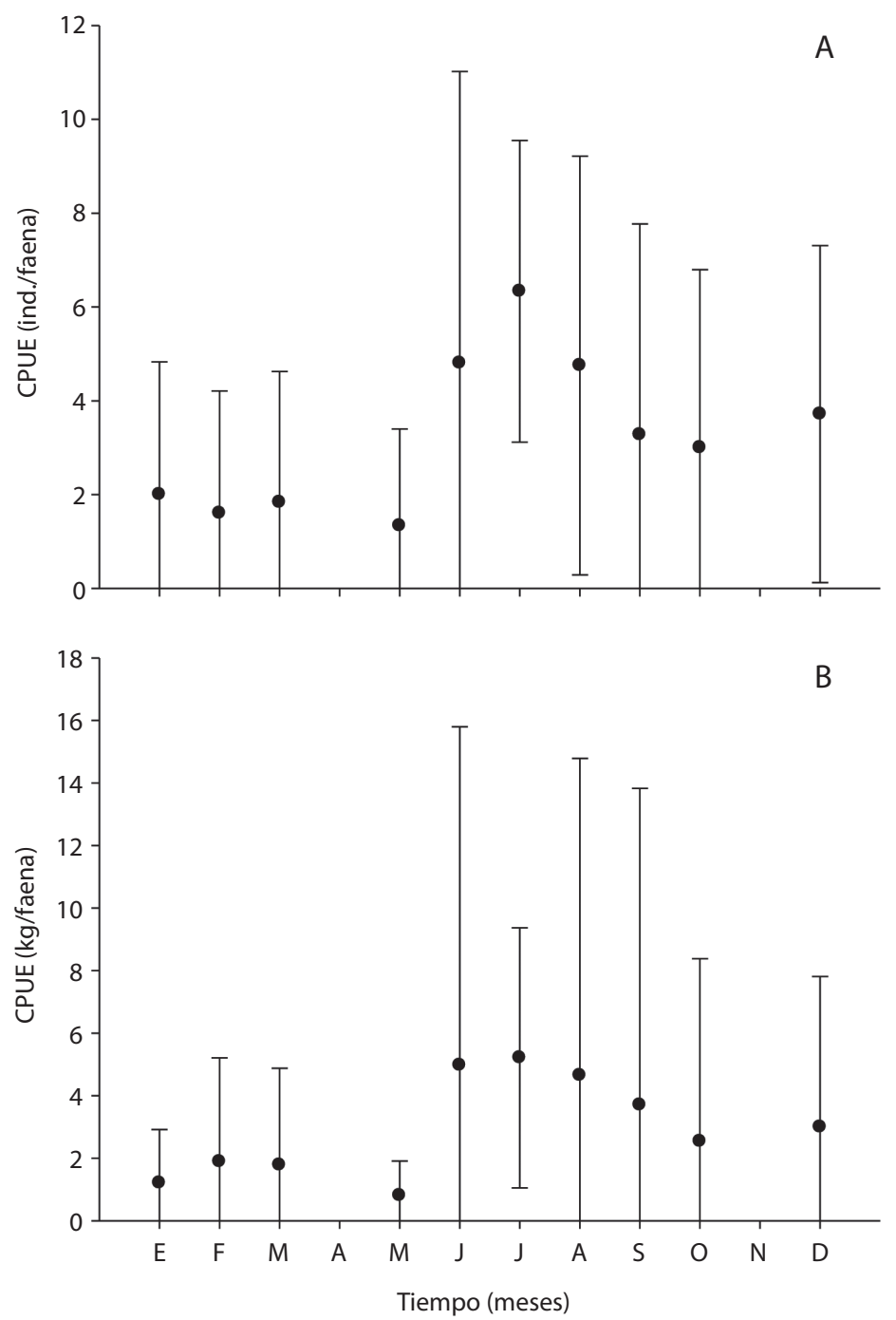

Fig. 2. (A) Promedio y desviación estándar mensual de la captura por unidad de esfuerzo (ind./faena). (B) CPUE (kilos/ faena) de Rhinobatos percellens, capturados por la pesquería artesanal de playa La Pared, Venezuela.

Fig. 2. (A) Monthly average and standard deviation of catch per unit effort (ind./trip). (B) CPUE (kg/trip) of Rhinobatos percellens captured by the artisanal fishery playa La Pared, Venezuela.

grávida con embriones cercanos a los $18 \mathrm{~cm}$ presentó ovocitos con talla máxima de $3.2 \mathrm{~cm}$ de diámetro.

Un total de 96 embriones con tallas entre $2.1-18.7 \mathrm{~cm}$ fueron analizados. La fecundidad máxima observada fue de cuatro embriones. El espectro de tallas más amplio se encontró en febrero y julio, mientras que las longitudes máximas se ubicaron en los mismos meses anteriores y en agosto (Fig. 5A).

Las hembras maduras fueron observadas durante todo el año, mientras que las grávidas estuvieron presentes en siete de los meses muestreados, siendo más abundantes en febrero, marzo y septiembre. Sin embargo, destaca su presencia durante todo el segundo semestre 

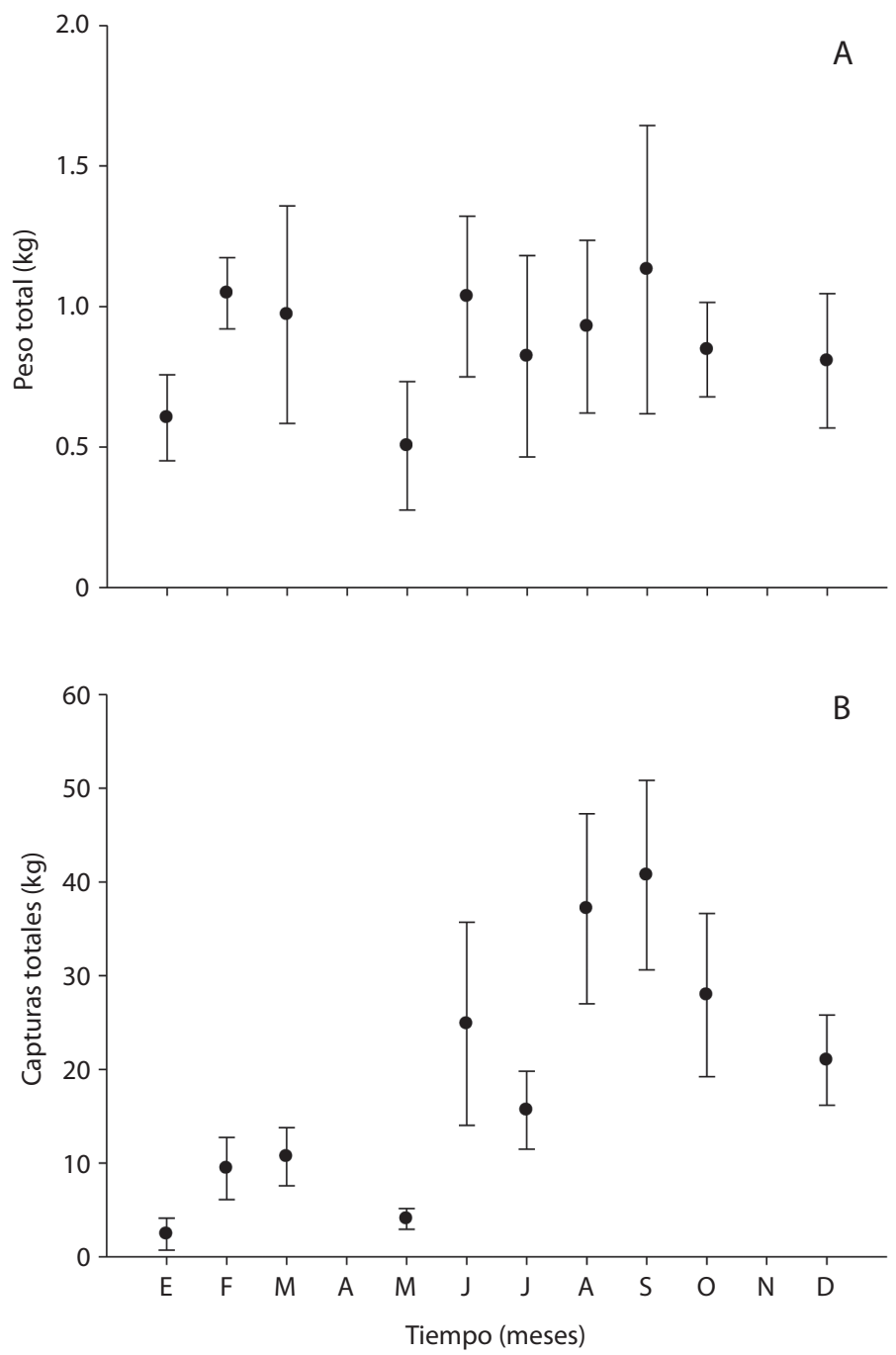

Fig. 3. (A) Peso promedio y desviación estándar de los individuos capturados. (B) Captura (kg) promedio y desviación estándar mensual de los ejemplares de Rhinobatos percellens capturados por la pesquería artesanal de playa La Pared, Venezuela.

Fig. 3. (A) Monthly average weight and standard deviation of individuals. (B) Monthly average caught and standard deviation of Rhinobatos percellens by the artisanal fishery of playa La Pared, Venezuela.

del año (julio-diciembre) (Fig. 5B). Todas las hembras grávidas presentaban óvulos en proceso de maduración en los ovarios. No se detectaron machos maduros durante el período febrero-mayo, mientras que a partir de junio siempre estuvieron presentes, en junio-julio no se encontraron individuos inmaduros en ese periodo (Fig. 5C).

\section{DISCUSIÓN}

Un indicativo de que una población de peces está siendo explotada de forma moderada, es el predominio de individuos maduros en las capturas (Cushing 1968). Este hecho ha sido observado en los desembarques de $R$. percellens de playa La Pared, ya que el $80.5 \%$ 

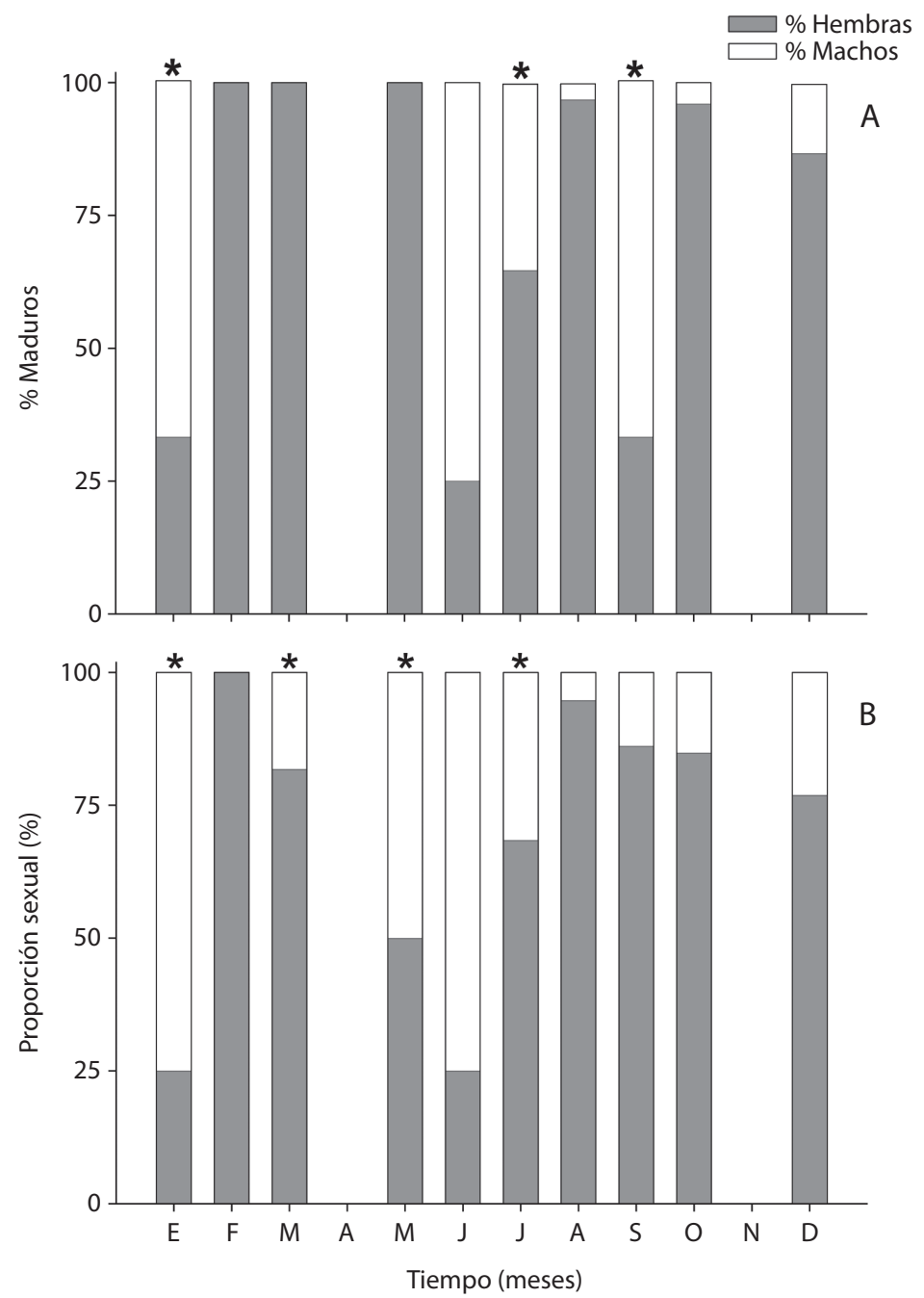

Fig. 4. (A) Proporción de hembras y machos maduros. (B) Proporción sexual mensual de Rhinobatos percellens capturado por la pesquería artesanal de playa La Pared, Venezuela. * No existen diferencias significativas.

Fig. 4. (A) Males and females mature proportions. (B) Monthly sex ratio of Rhinobatos percellens caught by the artisanal fishery of playa La Pared, Venezuela. * No significant differences.

de los individuos capturados se encontraban sexualmente maduros. No obstante, un $27 \%$ de estos ejemplares eran hembras grávidas, lo que mantiene en cierta forma una señal de alerta en la pesquería, puesto que se está afectando el potencial reproductivo de la especie. Esta presencia elevada de hembras grávidas en las capturas, también ha sido evidenciado en otras pesquerías; en Brasil se ha detectado hasta un $98 \%$ de hembras de $R$. horkelli capturadas en esta condición (Lessa \& Vooren 2007). Por esto, se sugiere tomar como tallas mínimas de captura, la longitud en la que el $100 \%$ de las hembras y machos se encuentran maduros (58 y $60 \mathrm{~cm}$, respectivamente), para asegurar que las hembras puedan aportar efectivamente su descendencia al stock.

Las hembras de $R$. percellens alcanzaron tamaños corporales superiores a los machos, condición similar a la observada para esta 
CUADRO 1

Estimados de a y b (Talla media de madurez sexual), de la ecuación logística para la proporción de machos y hembras maduros de Rhinobatos percellens capturado por la pesquería artesanal de playa La Pared, Venezuela

TABLE 1

Estimates of $\mathrm{a}$ and $\mathrm{b}$ (average size of sexual maturity), of the logistic equation for the proportion of mature males and females of Rhinobatos percellens captured by the artisanal fishery of playa La Pared, Venezuela

\begin{tabular}{|c|c|c|c|c|c|}
\hline \multirow{2}{*}{ Sexo } & \multirow{2}{*}{ Parámetro } & \multirow{2}{*}{ Estimado } & \multirow{2}{*}{ Error estándar } & \multicolumn{2}{|c|}{ Intervalos de confianza } \\
\hline & & & & $2.5 \%$ & $97.5 \%$ \\
\hline \multirow{2}{*}{ Machos } & $A$ & 0.2035 & 0.0682 & 0.0882 & 0.3632 \\
\hline & $b$ (L50) & 52.1722 & 1.782 & 46.8454 & 55.4781 \\
\hline \multirow{2}{*}{ Hembras } & A & 0.5770 & 0.1631 & 0.3161 & 0.9839 \\
\hline & $b(\mathrm{~L} 50)$ & 51.0394 & 1.1541 & 47.6374 & 52.8567 \\
\hline \multirow{2}{*}{ Ambos sexos } & A & 0.19375 & 0.0627 & 0.10816 & 0.38686 \\
\hline & $b(\mathrm{~L} 50)$ & 49.2399 & 1.7504 & 45.0125 & 52.8558 \\
\hline
\end{tabular}

especie en Colombia (Grijalba-Bendeck et al. 2008) y para $R$. horkelli en Brasil (Lessa \& Vooren 2007). La talla media de madurez sexual de las hembras fue inferior a la de los machos, lo que coincide con lo señalado para el Caribe Colombiano, aunque con longitudes inferiores tanto para hembras $(47.4 \mathrm{~cm})$ como para machos $(50.4 \mathrm{~cm})$ (Grijalba-Bendeck et al. 2008).

El mayor ejemplar encontrado en el presente estudio $(76.8 \mathrm{~cm})$, y el encontrado en Colombia $(73.5 \mathrm{~cm})$ (Grijalba-Bendeck et al. 2008), son inferiores a longitud máxima de $100 \mathrm{~cm}$ indicada hace unos 20 años por Cervigón et al. (1992). Linton \& Warner (2003) señalan que las disminuciones en las longitudes máximas pueden ser consideradas como el inicio de la sobreexplotación.

La ausencia de juveniles (con longitudes inferiores a $30 \mathrm{~cm}$ ), podría ser indicativo de que estos organismos pueden escapar de las redes, ya que éstas suelen tener una abertura superior a tres pulgadas. También existe la posibilidad de que frecuenten hábitats diferentes a los adultos y no se encuentren en las zonas de pesca. De hecho, en Brasil se ha comprobado que los recién nacidos de $R$. horkelli permanecen cercanos a costa durante todo el año, mientras que los adultos pueden migrar entre aguas profundas y someras, dependiendo de la época del año y la etapa reproductiva en la que se encuentren (Lessa \& Vooren 2007). Grijalba-Bendeck et al. (2008) encontraron ejemplares de $17 \mathrm{~cm}$, capturados con chinchorro playero que operan desde una distancia de $400 \mathrm{~m}$ hacia la costa.

La diferencia observada en la proporción sexual de $R$. percellens, podría indicar una segregación sexual en espacio y/o tiempo (Tresierra \& Culquichicón 1993). De hecho, el predominio de grupos compuestos por hembras grávidas solitarias, hembras maduras solitarias y machos inmaduros con hembras maduras, apoyaría esta teoría de segregación espacial. Sin embargo, en Colombia se han encontrado proporciones sexuales iguales (Grijalba-Bendeck et al. 2008).

Iguales proporciones sexuales en enero, marzo, mayo y julio, apuntan a que durante estos meses la cópula podría ser más intensa. Sin embargo, al comparar las proporciones sexuales únicamente tomando en cuenta individuos maduros, se observa que en marzo y mayo no se encontraron machos maduros, lo que reduce estos posibles picos de apareamiento a enero, julio y septiembre, meses en los que no se detectaron diferencias significativas en la proporción sexual de individuos maduros. No obstante, la presencia de embriones menores a $5 \mathrm{~cm}$ en el mes de julio, implicaría que existió cópula en los meses anteriores. 


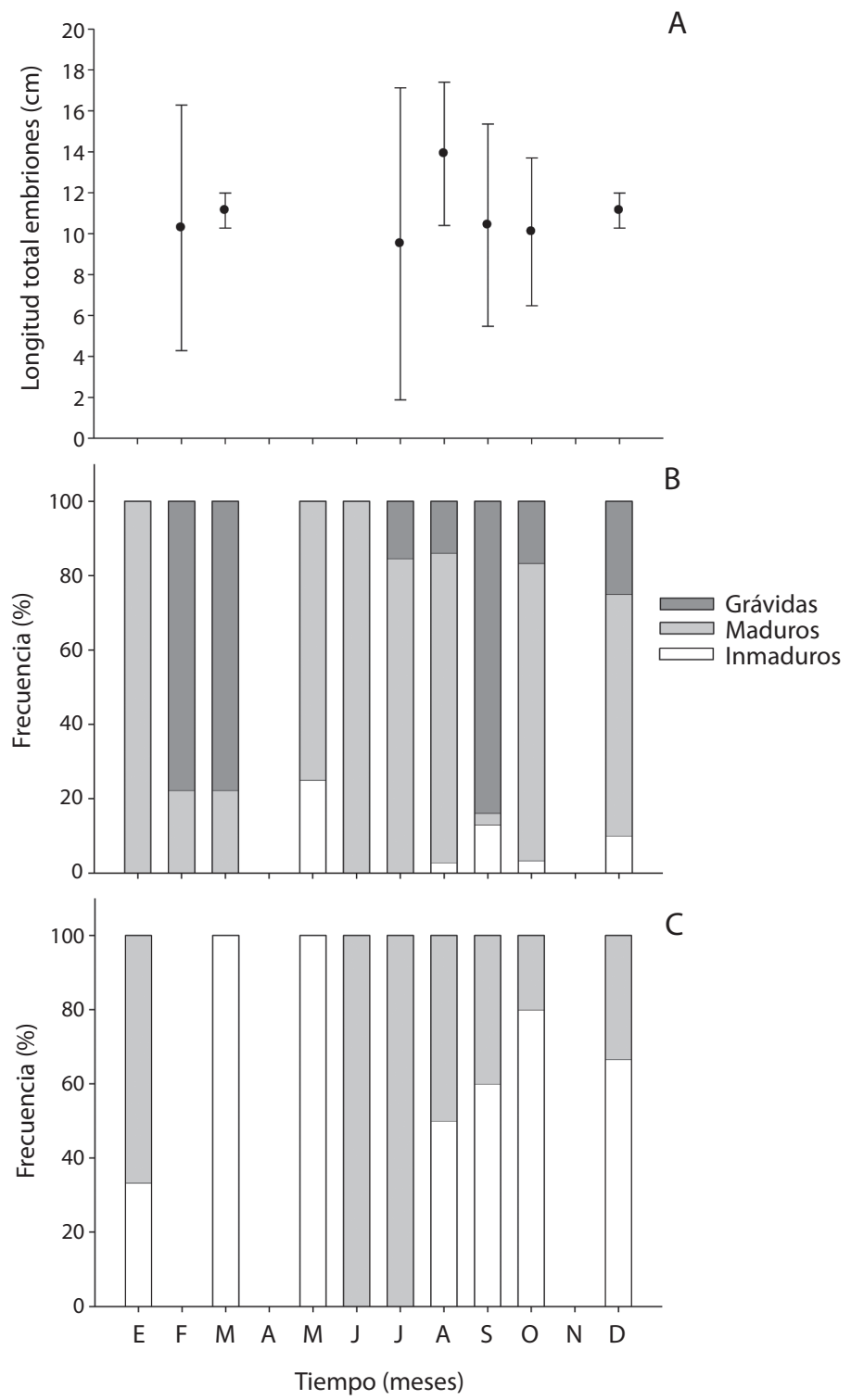

Fig. 5. (A) Promedio y desviación estándar mensual de las longitudes de los embriones de Rhinobatos percellens. (B) Frecuencia mensual de los diferentes estadios de madurez observados para hembras de Rhinobatos percellens. (C) Frecuencia mensual de los diferentes estadios de madurez observados para machos de Rhinobatos percellens capturados por la pesquería artesanal de playa la Pared, Venezuela.

Fig. 5. (A) Monthly average lengths and standard deviation of Rhinobatos percellens embryos. (B) Monthly frequency of different stages of maturation observed for females. (C) Monthly frequency of different stages of maturation observed for males of Rhinobatos percellens captured by the artisanal fishery of playa la Pared, Venezuela.

Adicionalmente, entre junio y diciembre siem- Grijalba-Bendeck et al. (2008), aunque ha pre se observó al menos una pareja de ejemplares maduros en las capturas.

Una fecundidad máxima de cuatro sido señalado que se pueden encontrar hasta seis embriones en una misma camada (Dahl 1971). Se sugiere un intervalo de talla de embriones, coincide con lo indicado por nacimiento de entre $17-20 \mathrm{~cm}$, en función de la 
talla máxima de embrión observada $(18.7 \mathrm{~cm})$, así como ejemplares libres con talla mínima de $17 \mathrm{~cm}$ (Grijalba-Bendeck et al. 2008), y una talla cercana al nacimiento reportada por Dahl (1971) de $20 \mathrm{~cm}$.

La observación de hembras grávidas con embriones en tallas cercanas al nacimiento y con ovocitos de gran diámetro, indica que, posiblemente estén listas para copular una vez que salen de la etapa de alumbramiento. Esta simultaneidad entre vitelogénesis de los óvulos y presencia de embriones uterinos ha sido observada también para $R$. percellens (Colombia), $R$. cemiculus y $R$. productus. No obstante, para estas especies se indica una única época reproductiva entre abril y agosto. Después de la expulsión de las crías, las hembras ovulan y copulan, y luego se mantienen en receso durante los meses restantes (Villavicencio 1993, Seck et al. 2004, Grijalba-Bendeck et al. 2008). De manera contraria, este estudio no permite afirmar ningún patrón marcado de alguna de las etapas del periodo reproductivo, sino que se observaron hembras grávidas y embriones de diferentes tallas durante prácticamente todo el año, lo que parece indicar que no hay una sincronía marcada para la cópula, periodo de gestación y alumbramiento.

Grijalba-Bendeck et al. (2008) encuentran épocas marcadas de nacimientos entre febreromarzo y octubre-noviembre, meses del año con mayor productividad en el Caribe colombiano. En Venezuela también se observan dos posibles épocas con alumbramiento un poco más intenso que el resto del año, evidenciado por las tallas cercanas al nacimiento de los embriones observados en febrero, junio y julio. Sin embargo, la especie parece mostrar una reproducción continua, por las elevadas proporciones de hembras grávidas y variabilidad de tallas de embriones a lo largo del año. Con base a esta última observación, y considerando un periodo de gestación de unos cuatro meses, sugerido por Grijalba-Bendeck et al. (2008), se pueden realizar progresiones hipotéticas de las longitudes de los embriones hasta el rango de talla cercano al nacimiento, lo cual permite deducir que se pueden producir alumbramientos durante prácticamente todos los meses del año. No obstante, a pesar de que no se detecta una marcada sincronía reproductiva entre las diferentes hembras, probablemente estas pueden efectuar dos ciclos reproductivos al año.

Esta posible reproducción continua de la especie podría verse favorecida por el afloramiento de aguas ricas en nutrientes, producto de la surgencia costera y las descargas de ríos como el Orinoco (Gómez 1996), que favorecen el mantenimiento de altas densidades de fito y zooplancton, así como abundante materia orgánica particulada, que permiten el desarrollo de extensas comunidades de moluscos bivalvos (Cervigón 2005), las cuales proveen refugio a comunidades de crustáceos (Hernández-Ávila et al. 2007); que representan el principal ítem alimenticio de $R$. percellens (Polo-Silva \& Grijalba-Bendeck 2008, Bornatowsky et al. 2010), lo que implica la disponibilidad permanente de alimento para la especie.

Un elevado rendimiento en las capturas registradas para agosto y septiembre puede estar relacionado con condiciones idóneas para la navegación durante esos meses del año, lo que probablemente haya provocado un mayor esfuerzo de pesca y con ello mayores capturas de la especie. El hecho de que exista una gran cantidad de faenas donde no se capturó ningún ejemplar, podría ser explicado por el uso de zonas de pesca donde la especie no habita o a posibles reducciones en la abundancia de la especie. Sin embargo, no existen datos históricos de índices de abundancia, que permitan hacer alguna comparación de las capturas en el tiempo. No obstante, existen cifras que demuestran que la producción general de $R$. percellens para el estado Nueva Esparta, se redujo de $8500 \mathrm{~kg}$ capturados en 2006 a 7 $300 \mathrm{~kg}$ en el 2007 (Marval \& Cervigón 2009).

Es importante señalar que todos los botes muestreados utilizaron diversos artes de pesca en diferentes épocas del año, dependiendo de las variaciones en la abundancia temporal de los diferentes recursos que explotan. Por ejemplo, en abril se detectó gran abundancia recursos pelágicos (pequeños atunes); mientras que en noviembre hubo un desplazamiento de la flota a 
zonas de mayor profundidad para capturar otros elasmobranquios (Mustelus norrisi, M. canis, Squalus spp. y Squatina dumeril), lo que produjo una disminución del esfuerzo hacia los recursos costaneros demersales, por lo que durante dichos meses no se capturaron ejemplares de $R$. percellens. Esta manera de alternar los artes de pesca debe ser considerada como una excelente herramienta de manejo pesquero aplicada por los pescadores de playa La Pared, ya que rotan las zonas de pesca, dándo descanso a algunos recursos, mientras extraen otros.

En la mayoría de los países donde las capturas no pueden ser controladas, la implementación de áreas marinas protegidas proveen una efectiva y rápida medida de conservación (Hixon et al. 2001). La especie ha sido observada en numerosas ocasiones en los desembarques de los pescadores de los alrededores de la isla de Cubagua, zona que por su alto valor histórico (Otte 1977, Cervigón 1997, 1998) tiene gran potencial para ser decretada como área marina protegida.

Se recomienda evaluar la reproducción de la especie durante varios años consecutivos para poder detectar su variabilidad temporal, ya que el éxito de los sucesos reproductivos puede variar anualmente en respuesta a condiciones oceanográficas, especialmente en ecosistemas dominados por fenómenos de surgencia costera (Parrish et al. 1981).

Los elevados porcentajes de hembras grávidas presentes en las capturas, así como el aumento de las flotas pesqueras y la demanda de productos marinos, como consecuencia del constante crecimiento de la población venezolana, pueden ser consideradas las principales amenazas para la especie. Es importante implementar una medida de regulación pesquera que incluya talla mínima de captura en función de la talla de madurez señalada, así como campañas educativas que destaquen la importancia de devolver las hembras grávidas al agua.

\section{AGRADECIMIENTOS}

Al Instituto Nacional de Investigaciones Agrícolas (INIA) y Asdrúbal Lárez por su valiosa colaboración en la logística de muestreos. A la comunidad de pescadores de playa La Pared por su gentileza al dejarnos analizar sus capturas. Paula Young por la revisión del resumen en inglés. A los árbitros anónimos por sus acertadas sugerencias.

\section{RESUMEN}

Rhinobatos percellens es capturada en Venezuela por la flota artesanal que opera con redes de fondo. Está clasificada por la IUCN como "casi amenazada", y no existen estudios sobre su biología o pesquería en el país. Por ello, se analizaron desembarques de la pesca artesanal de playa la Pared (isla de Margarita) durante enero-diciembre 2007. A cada ejemplar capturado se le determinó la longitud total, sexo y madurez. Fueron analizados 210 ejemplares, 159 hembras y 51 machos. El $81 \%$ de los ejemplares se encontraban maduros y un $27 \%$ de este grupo correspondió a hembras grávidas. La talla media de madurez sexual quedó ubicada en 51 y $52 \mathrm{~cm}$ para hembras y machos, respectivamente. Se detectaron hembras maduras todo el año, y hembras grávidas en siete meses. Fueron analizados 96 embriones con tallas entre $2-19 \mathrm{~cm}$, siendo la fecundidad máxima cuatro embriones. La variación de las longitudes de los embriones entre los meses del año indican dos picos máximos de alumbramiento al año pero no existe una sincronía en la cópula ya que la especie se reproduce prácticamente todo el año. Se recomienda elaborar regulaciones pesqueras que incluyan una talla mínima de captura en función de la talla de madurez señalada y liberar las hembras grávidas al agua.

Palabras clave: Elasmobranquio, batoideo, pez guitarra, Caribe.

\section{REFERENCIAS}

Aparicio, R. 2003. Revisión de las características oceanográficos de la plataforma nororiental de Venezuela, p. 171-205. In P. Freón \& J. Mendoza (eds.). La sardina (Sardinella aurita), su medio ambiente y explotación en el oriente de Venezuela. Institut de Reserche pour le Développement. Collection Colloques et seminaries, Paris.

Bizzarro, J., W. Smith, F. Márquez-Farías \& R. Hueter. 2007. Artisanal fisheries and reproductive biology of the golden cownose ray, Rhinoptera steindachneri Evermann and Jenkins, 1891, in the northern Mexican Pacific. Fish. Res. 84: 137-146.

Bornatowsky, H., M. Castro \& L. Costa. 2010. Feeding of guitarfish Rhinobatos percellens (Walbaum, 1972) (Elasmobranchii, Rhinobatidae), the target of artisanal fishery in southern Brazil. Brazil. J. Oceanogr. 58: $45-51$. 
Casper, B. \& G. Burgess. 2004. Rhinobatos percellens. En: IUCN 2010. IUCN Red List of Threatened Species. Version 2010.4. (www.iucnredlist.org).

Cervigón, F., R. Cipriani, W. Fischer, L. Garibaldi, M. Hendrickx, A. Lemus, R. Márquez, J. Poutiers, G. Robaina \& B. Rodríguez. 1992. Fichas FAO de identificación de especies para los fines de la pesca. Guía de campo de las especies comerciales marinas y de aguas salobres de la costa septentrional de Sur América, FAO, Roma, Italia.

Cervigón, F. 1997. Cubagua 500 años. Fundación Museo del Mar, Caracas, Venezuela.

Cervigón, F. 1998. Las perlas en la historia de Venezuela. Fundación Museo del Mar, Caracas, Venezuela.

Cervigón, F. \& A. Alcalá. 1999. Los peces marinos de Venezuela, V. Fundación Museo del Mar, Caracas, Venezuela.

Cervigón, F. 2005. La ictiofauna marina de Venezuela: una aproximación ecológica. Bol. Inst. Oceanogr. 44: 3-28.

Cheung, W, T. Pitcher \& D. Pauly. 2005. A fuzzy logic expert system to estimate intrinsic extinction vulnerabilities of marine fishes to fishing. Biol. Conserv. 124: 97-111.

Conrath, C. 2004. Reproductive biology, p. 133-164. In J. Musick \& R. Bonfil (eds.). Elasmobranch fisheries management techniques, APEC Secretariat, Singapore.

Cushing, D. 1968. Fishery biology. A study in population dynamics, University of Wisconsin, Londres, Inglaterra.

Dahl, G. 1971. Los peces del Norte de Colombia. Instituto de Desarrollo de los Recursos Naturales Renovables, Bogotá, Colombia.

Froese, R., M. Palomares \& D. Pauly. 2002. Estimation of Life-History Key Facts, Resilience to fishing pressure. Fish Base (Consultado: 10 diciembre 2011, www. fishbase.org).

Gómez, A. 1996. Causas de la fertilidad marina en el nororiente de Venezuela. Interciencia 21: 140-146.

González, L., N. Eslava \& F. Guevara. 2006. Catálogo de la pesca artesanal del Estado Nueva Esparta, Venezuela, Instituto de Investigaciones Científicas, Universidad de Oriente, Boca de Río, Venezuela.

Grijalba-Bendeck, M., A. Acero \& E. González. 2008. Biología reproductiva de Rhinobatos percellens (Walbaum, 1972) (Batoidea: Rajiformes) en el Caribe colombiano. Rev. Biol. Mar. Oceanogr. 43: 469-481.

Hernández-Ávila, I., A. Gómez, C. Lira \& L. Galindo. 2007. Benthic decapod crustaceans (Crustacea: Decapoda A.) of Cubagua Island, Venezuela. Zootaxa 1557: 33-45.
Hixon, M., P.D. Boersma, M. Hunter Jr., F. Micheli, E. Norse, H. Possingham \& P. Snelgrove. 2001. Oceans at risk: research priorities in marine conservation biology, p. 125-154. In M. Soulé \& G. Orians (eds.). Conservation biology: Research priorities for the next decade, Island, Washington, EEUU.

Iriarte, L. 1997. Embarcaciones, artes y métodos de pesca del estado Nueva Esparta. Fundación La Salle de Ciencias Naturales. Distrito Federal, Caracas, Venezuela.

Leal, R. 2008. Análisis de la erosividad de la lluvia en la isla de Margarita (Venezuela) a través de datos de precipitación horaria. Invest. Geogr. 44: 167-185.

Linton, D. \& G. Warner. 2003. Biological indicators in the Caribbean coastal zone and their role in integrated coastal management. O. Coast. Manag. 46: 261-276.

Lessa, R. \& C. Vooren. 2007. Rhinobatos horkelii. IUCN 2010. IUCN Red List of Threatened Species. Version 2010.4. (Consultado: 10 diciembre 2011, www.iucnredlist.org).

Ley de Pesca y Acuicultura. 2008. Artículo N²3. Decreto No 5930 Publicado en Gaceta Oficial de la República Bolivariana de Venezuela $N^{\circ} 5877$ de 14 de marzo de 2008.

Marval, J. \& F. Cervigón. 2009. Actividad pesquera en el estado Nueva Esparta. Fundación Museo del Mar, Ex Libris, Boca de Río, Venezuela.

Otte, E. 1977. La perla del Caribe. Nueva Cádiz de Cubagua. Fundación Boulton, Caracas, Venezuela.

Parrish, R., C. Nelson \& A. Bakun. 1981. Transport mechanisms and reproductive success of fishes in the California Current. Biol. Oceanogr. 1: 175-203.

Polo-Silva, C. \& M. Grijalba-Bendeck. 2008. Espectro trófico de la raya guitarra Rhinobatos percellens (Walbaum, 1792) (Elasmobranchii: Rhinobatidae) en Santa Marta, Caribe, Colombia. Mem. Soc. Cs. Nat. Salle 169:21-33.

Seck, A., T. Diatta, M. Diop, O. Guélorget, D. Reynaud \& C. Capapé. 2004. Observations on the reproductive biology of the blackchin guitarfish, Rhinobatos cemiculus E. Geoffroy Saint-Hilaire, 1817 (Chondrichthyes, Rhinobatidae) from the coast of Senegal (Eastern Tropical Atlantic). Sc. Gerundensis 27: 19-30.

Tresierra, A. \& Z. Culquichicón. 1993. Biología pesquera. Consejo Nacional de Ciencia y Tecnología, Trujillo, Perú.

Villavicencio, C. 1993. Biología reproductiva de Rhinobatos productus (Pisces: Rhinobatidae), en bahía Almejas, Baja California Sur, México. Rev. Biol. Trop. 41: 777-782. 